\title{
WORKFORCE Newly graduated doctors' experiences of conducting medical ward rounds alone: A regional cross-sectional study
}

\author{
Authors: Jessica Pearce, ${ }^{\mathrm{A}}$ Sameet Govan, ${ }^{\mathrm{B}}$ Anna Harlinska, ${ }^{\mathrm{C}}$ Rebecca Tremain, ${ }^{\mathrm{D}}$ Sareena Gajebasia ${ }^{\mathrm{E}}$ and Melody Redman ${ }^{\mathrm{F}}$
}

Newly graduated doctors may be expected to conduct ward rounds alone, yet studies exploring this are limited. A regional cross-sectional study was undertaken to explore foundation year 1 doctors' (FY1s) experiences of conducting ward rounds alone; all 289 FY1s on medical rotations in Yorkshire and the Humber Foundation School were invited to participate in an online survey in November 2016. Thirty-four percent $(n=98)$ responded. The majority $(62 \%, n=61)$ of respondents reported conducting the daily ward round alone (without a more senior doctor present) two or more times a week. However, $56 \%$ $(n=55)$ reported that they had never received teaching on ward round conduct and only $7 \%(n=7)$ reported feeling prepared for conducting ward rounds alone at the start of their medical rotation. FY1-led ward rounds are a regular occurrence yet training is not commonplace; widespread, early training should be considered to prepare future doctors for their role.

KEYWORDS: Ward round, foundation year 1, leadership, medical education, patient care

\section{Introduction}

Ward rounds are fundamental to the care of medical inpatients, and newly graduated doctors are a key part of their delivery. The Royal College of Physicians describe ward rounds as ' $a$ complex clinical process during which the clinical care of hospital inpatients is reviewed'. In addition to medical knowledge, this process requires a range of skills, including technical competency, communication, teamwork and patient management. ${ }^{2}$ Although these key skills are covered in medical training, specific teaching on the complex process of ward round conduct is not commonplace; a pre-training survey by Powell et al revealed that only $9 \%$ of 56

Authors: ${ }^{\text {A }}$ core medical trainee, Leeds Teaching Hospitals NHS Trust, Leeds, UK; ${ }^{B}$ general practice specialty trainee year 1, The Mid Yorkshire Hospitals NHS Trust, Wakefield, UK; ${ }^{C}$ emergency medicine trainee, Hull and East Yorkshire Hospitals NHS Trust, Hull, UK; D trust grade, Hull and East Yorkshire Hospitals NHS Trust, Hull, UK; ${ }^{\mathrm{E}}$ core medical trainee, Hull and East Yorkshire Hospitals NHS Trust, Hull, UK; Facademic clinical fellow paediatrics, Sheffield Children's NHS Foundation Trust, Sheffield, UK medical students and $27 \%$ of 30 foundation doctors had prior training in ward round conduct. ${ }^{3}$ Furthermore, competency in this area is not a specific requirement of UK medical graduates ${ }^{4}$ or foundation doctors ${ }^{5}$ (those on a two-year training programme following graduation from medical school called 'The Foundation Programme'). A study of final-year medical students' ward round skills showed deficits in their ability to perform focused physical examinations, review charts, prescribe and document the findings of the ward round. ${ }^{2}$

Previous quality improvement work undertaken by Pearce, Redman and Gajebasia et al revealed that $85 \%(n=16)$ of 19 foundation year 1 doctors (FY1s) in one NHS trust (consisting of two district general hospitals which host FY1s) reported conducting medical ward rounds alone twice or more each week. ${ }^{6}$ Furthermore, $89 \%(n=17)$ of respondents reported feeling unprepared for conducting ward rounds alone, ${ }^{6}$ a finding supported by two other studies that demonstrated low levels of confidence for ward round conduct and related skills in medical students and foundation doctors. ${ }^{5,7}$ The high frequency of FY1-led ward rounds ${ }^{6}$ and lack of self-perceived preparedness ${ }^{5-7}$ is concerning, especially given the lack of prior training ${ }^{5}$ and skill deficit ${ }^{2}$ identified in this area. The studies exploring this area to date are limited in number, and are predominantly small, local studies, but they clearly highlight the need for larger scale exploration into newly graduated doctors' experiences of conducting ward rounds alone.

This study aimed to explore the experiences of FY1s conducting ward rounds alone in more depth and across a wider geographical area in order to inform changes to training and support for future doctors, and subsequently improve patient care on the wards.

\section{Methods}

An online survey was designed to explore FY1s' experiences of conducting ward rounds alone across Yorkshire and the Humber Foundation School (YHFS), the fourth largest of 20 foundation schools in the UK. ${ }^{8}$ All $289 \mathrm{FY} 1 \mathrm{~s}$ on medical rotations in YHFS during August to December 2016 were invited to participate. At the time of the study, YHFS included three locality schemes and 13 NHS trusts. FY1s were invited to participate via email, e-portfolio messages, group messaging on a smartphone messaging platform, social media platforms and word of mouth. The survey was open for the final 14 days of their medical rotation. 
FY1s were asked to reflect on their experiences of conducting ward rounds alone during their first medical rotation. The survey focused on one rotation to minimise recall bias; the first rotation was chosen to provide a valuable insight into the important transition from medical student to doctor. Medical rotations were chosen to minimise possible confounding variables between ward rounds of different hospital specialties.

Demographic data were collected regarding participants' subspeciality and locality scheme within YHFS. Participants were asked whether they had received teaching on how to conduct a ward round, how many times per week they conducted the daily ward round alone (without a more senior doctor present on the ward round), and how many times per week a higher trainee or consultant conducted the daily ward round (for comparison). The participants were also asked which decisions regarding patient care they were able to make without additional delay when they conducted the ward round alone compared with when their senior colleagues did, and which aspects of care they regularly considered during ward rounds they conducted alone, to gain an insight into the impact FY1-led ward rounds may have on patient care. These questions required FY1s to select from a list of preprepared options.

The participants were also asked to rate their agreement with a series of six statements via a five-point Likert scale ${ }^{9}$ from 'Strongly agree' to 'Strongly disagree'. The first three statements related to their experiences of conducting ward rounds alone (whether they felt prepared at the start and end of their rotations respectively, and whether they felt supported when conducting ward rounds alone). The final three related to their views of FY1-led ward rounds (whether conducting ward rounds alone was a valuable learning experience, whether FY1s should receive formal teaching on how to conduct a ward round, and whether FY1s should conduct medical ward rounds alone).

The survey concluded with optional free text comments, which provided an opportunity for participants to discuss their experiences and views in more depth. Thematic analysis was undertaken on these qualitative data, the findings of which will be prepared for separate publication.

Participation was voluntary and participants gave their implied consent by completing the online survey. Research and development approval was attained from the NHS Health Research Authority (HRA) on behalf of all NHS trusts within the region. Ethical approval was granted by the Hull York Medical School Ethics Committee.

\section{Results}

Thirty-four percent ( $n=98$ ) of the $289 \mathrm{FY} 1 \mathrm{~s}$ on medical rotations in YHFS completed the survey. Respondents were spread representatively across the three locality schemes: West Yorkshire, $39 \%(n=38)$; South Yorkshire, $32 \%(n=31)$; and North Yorkshire and East Coast, 29\% ( $n=29)$. Respondents were from a range of medical specialties (Fig 1), with the largest proportion working in either elderly or respiratory medicine $(33 \%, n=32$ and $21 \%, n=20$ respectively).

\section{Prior teaching}

Fifty-six percent ( $n=55$ ) of FY1s reported that they had never received teaching on ward round conduct, while $28 \%(n=27)$ of respondents received teaching during medical school, $23 \%(n=23)$

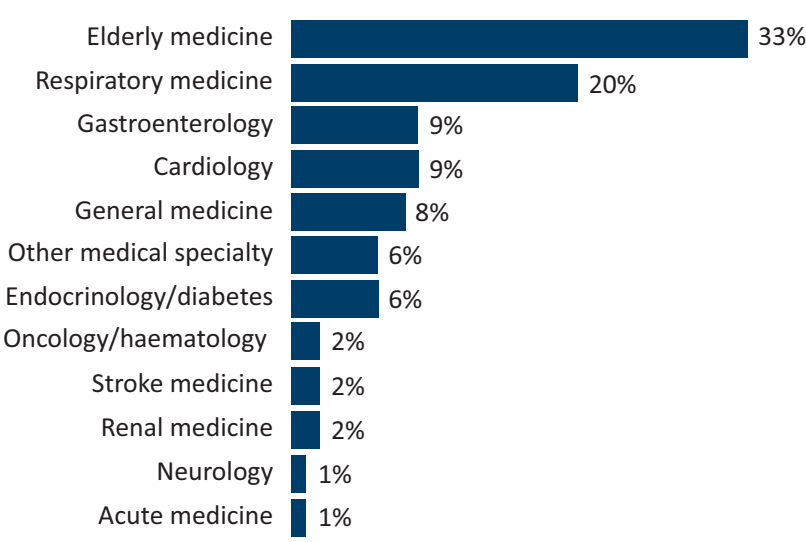

Percentage of FY1s

Fig 1. Breakdown of respondents by medical specialty. 'Which medical specialty is your rotation primarily based? - select best fit.

during the national shadowing period, and $16 \%(n=16)$ after starting work (some FY1s received teaching at more than one of these times).

Frequency of FY1-led and higher trainee/consultant-led ward rounds

The majority of respondents $(62 \%, n=61)$ reported conducting the daily ward round alone twice a week or more often (Fig 2).

When asked how often a higher trainee (ST3+/registrar) or a consultant conducted the ward round, $65 \%(n=64)$ of FY1s said this happened three times a week or less, $17 \%(n=17)$ four times a week and $17 \%(n=17)$ five times a week.

\section{FY1-led decisions without delay}

FY1s were asked to select from a list which decisions they were able to make without additional delay when conducting the ward round alone compared with when a senior doctor conducted the ward round. The proportion of FY1s selecting each of the responses are outlined in Fig 3.

\section{Aspects of care considered by FY1s}

Respondents were asked to select from a list which aspects of care they regularly considered when conducting the ward round alone. Findings are displayed in Fig 4.

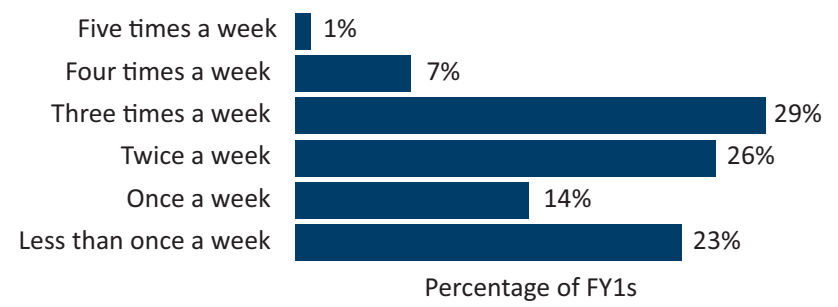

Fig 2. Frequency with which FY1s conducted the daily ward round alone. "During this rotation, on average, how many times per week did you conduct the daily ward round alone (ie without a more senior doctor present on the ward round)?' 


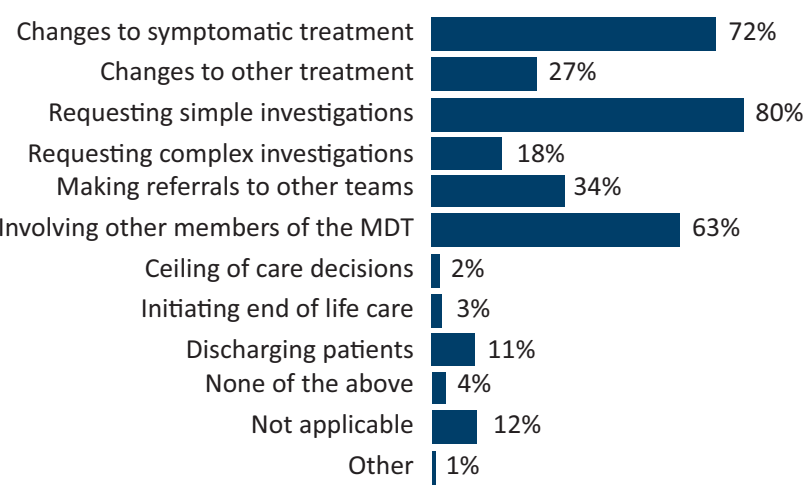

Percentage of FY1s

Fig 3. Percentage of FY1s able to make each decision without additional delay. 'On the days that you conducted the ward round alone, which of the following decisions were you able to make without additional delay (compared to when a senior doctor conducted the ward round)?'

\section{Self-reported preparedness}

Seven percent $(n=7)$ of FY1s reported feeling prepared for conducting ward rounds alone at the start of their medical rotation, compared with $64 \%(n=63)$ at the end. In contrast, $84 \%(n=82)$ felt unprepared at the start compared with $10 \%(n=10)$ at the end (see Fig 5).

\section{Perceived support}

Thirty-eight percent ( $n=37$ ) of respondents agreed or strongly agreed that they felt supported while conducting ward rounds alone, while $28 \%$ ( $n=28$ ) disagreed or strongly disagreed, and $34 \%(n=33)$ neither agreed nor disagreed.

\section{Educational value}

Sixty-five percent $(n=64)$ of respondents agreed or strongly agreed that conducting ward rounds alone as an FY1 is valuable for learning, while $11 \%(n=11)$ neither agreed nor disagreed, and $24 \%$ $(n=23)$ disagreed or strongly disagreed.

\section{Need for teaching}

Most respondents $(92 \%, n=90)$ agreed or strongly agreed that FY1s should receive formal teaching on how to conduct a ward

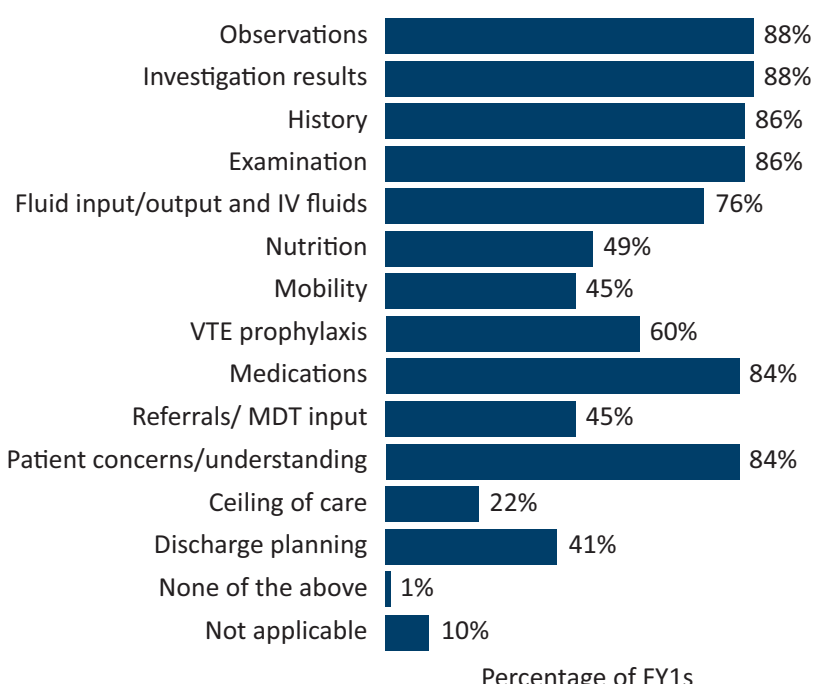

Percentage of FY1s

Fig 4. Percentage of FY1s that reported regularly considering these aspects of care when conducting the ward round alone. "Which of the following do you regularly consider when conducting the ward round alone?

round before starting work, while $6 \%(n=6)$ neither agreed nor disagreed, and $2 \%(n=2)$ disagreed.

\section{Overall view}

Thirty-five percent $(n=34)$ agreed or strongly agreed that FY1s should conduct medical ward rounds alone, $25 \%(n=25)$ neither agreed nor disagreed, and 40\% $(n=39)$ disagreed or strongly disagreed.

\section{Discussion}

\section{Key findings}

FY1-led ward rounds on medical wards in YHFS are common with $62 \%$ ( $n=61$ ) of FY1s reporting conducting ward rounds alone two or more times a week, yet only $7 \%(n=7)$ felt prepared for this task at the start of their medical rotation. At the end of the medical rotation there was some improvement; $64 \%(n=63)$ of FY1s felt prepared to conduct a ward round alone. However, only $38 \%$ $(n=37)$ of FY1s felt supported when conducting ward rounds alone.

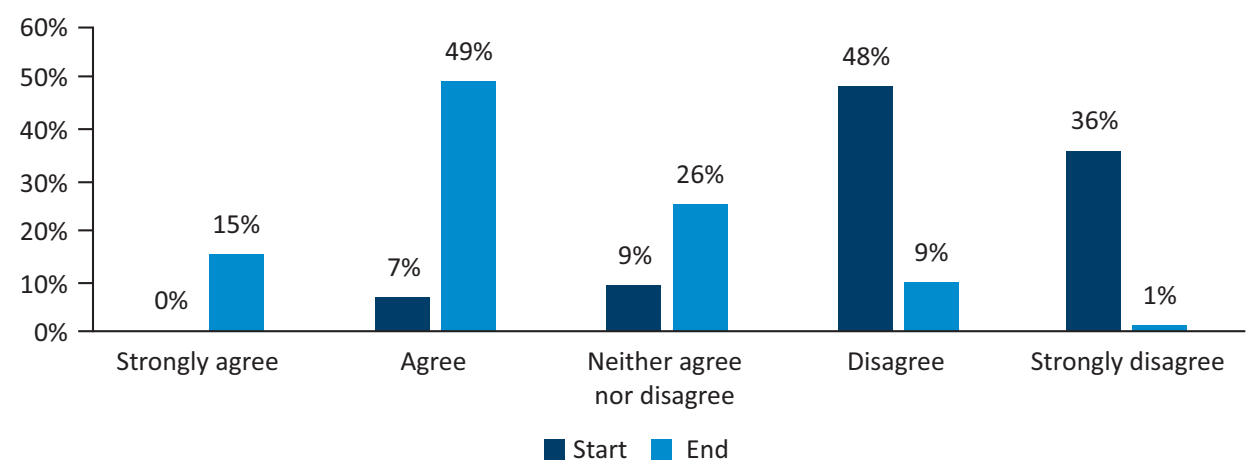

Fig 5. Percentage of FY1s reporting feeling prepared for conducting ward rounds alone at the start and end of their medical rotations, respectively. 'At the start/end of my medical rotation, I felt prepared for conducting ward rounds alone.' 
Most respondents $(92 \%, \mathrm{n}=90)$ agreed that $\mathrm{FY} 1 \mathrm{~s}$ should have formal teaching on how to conduct a ward round before starting work, but $56 \%(n=55)$ had never received teaching on how to conduct a ward round at the time of the survey. Overall opinions on whether FY1s should conduct ward rounds alone were mixed.

\section{Strengths and limitations of the study}

This study used an online survey to explore FY1s' experiences of conducting medical ward rounds alone within YHFS, one of the largest foundation schools in the UK, ${ }^{8}$ in order to maximise the external validity of the findings. The response rate was lower than the study target of $40 \%$, despite a 2 -week completion window and regular reminders sent to FY1s across a range of platforms, likely due to survey fatigue and other requirements and priorities of FY1s at the time of transition between rotations. However, $34 \%$ remains acceptable and allows reasonable generalisability to the experiences of FY1s on medical rotations elsewhere. The nature of self-reporting makes some degree of reporter and recall bias inevitable, with responses possibly being skewed towards negative experiences, but there was an attempt to minimise these biases by inviting all FY1s on medical rotations and asking them to reflect on only their most recent rotation. While an observational study might have improved the internal validity by reducing bias and providing more accurate quantification of some of the variables (such as the frequency of FY1-led and senior-led ward rounds, and the aspects of care considered and decisions made without delay during FY1-led ward rounds), it would not be practicable to conduct a true observational study across such a wide geographical area and therefore the generalisability of such a study would be limited. This study specifically enquired how many times per week FY1s 'conducted the daily ward round alone' as well as how often a senior doctor (registrar or consultant) did so, to provide an idea of how often the patients were being reviewed by a senior doctor. However, it must be recognised that the input of other colleagues (foundation year 2 doctors, core medical trainees etc) is not represented in the data, and it does not necessarily allow full appreciation of the complexities of the division of labour within the medical team. The first rotation as a newly graduated doctor is likely to be the most challenging time for FY1s to undertake ward rounds alone, introducing selection bias and limiting the generalisation of the results to FY1s on medicine during their second and third rotations, but exploring their experiences at this time gives a valuable insight into FY1s' experiences of conducting medical ward rounds alone when first starting as a new doctor.

\section{Comparison with previous work}

These findings reinforce previous data demonstrating that FY1s are regularly conducting ward rounds alone, ${ }^{6}$ but the proportion of FY1s reporting conducting ward rounds alone twice or more each week in this study was slightly lower than in the previous study ${ }^{6}$ ( $62 \%$ vs $84 \%$ respectively). One potential explanation could be that the previous study was conducted in two small district general hospitals (DGHs) ${ }^{6}$ whereas this study was conducted across YHFS, encompassing both DGHs and tertiary centres. It also supports previous studies demonstrating low levels of confidence and self-reported preparedness for ward round conduct. ${ }^{5-7}$ Less than half of those surveyed had received prior training in ward round conduct, which is in keeping with the previously described findings of Powell et al. ${ }^{5}$ This study in YHFS is the largest of its kind, covers a wide geographical area and encompasses both DGHs and tertiary centres giving stronger external validity.

\section{Meaning of the study}

FY1s on medical rotations in YHFS are regularly conducting ward rounds alone, but it is unclear whether this is planned or an unintended consequence of staffing issues or allocation of senior doctors' time. The majority of FY1s believed that conducting ward rounds alone was valuable for their learning, however there was a strong consensus among FY1s that they feel underprepared and under supported when conducting ward rounds alone, and that they should receive formal teaching on this topic before starting work. To some degree, these findings may be influenced by a lack of confidence at this early stage in training, but the results provide a strong argument for introducing training in ward round conduct for future doctors before they start work to prepare them for their future role. As previously discussed, ward rounds are a complex clinical process ${ }^{1}$ requiring a range of knowledge, skills and competencies; ${ }^{2}$ dedicated teaching is required to bring this learning together. There is a growing body of evidence to support the role of simulation and observed practice in teaching ward round competencies. ${ }^{5,10-13}$ A simple aide-memoire outlining aspects of care to consider on ward rounds has also been demonstrated to be of value to provide a framework for experiential learning on the wards. ${ }^{6}$

As might be expected, there were a number of decisions that the majority of FY1s could not make without delay, such as requesting complex investigations and those surrounding discharge and end-of-life care, indicating the possible implications of regular FY1-led ward rounds on patient care. These findings highlight the importance of having systems in place to ensure that timely senior support is available to FY1s at all times, both to improve patient care and to improve the experience and educational value for FY1s. Policy makers and hospital managers should plan work schedules to ensure that senior-led ward wards occur with adequate frequency and that senior support is available to FY1s at all times when they are expected to conduct ward rounds alone. Self-reported preparedness among FY1s was particularly low at the start of the rotation, suggesting that senior support is particularly important at this time, but improved drastically towards the end, demonstrating that the experience gained is valuable and that FY1s may need less support and be able to take on more responsibility in conducting the ward round towards the end of their first rotation and in subsequent posts. Senior doctors should ensure that FY1s on their team are working within their competencies and are adequately supervised at all times. Opinions on whether FY1s should conduct ward rounds alone were mixed, but may change if improvements in training and support for FY1s doing so were introduced.

In response to this work, a motion was raised and accepted into national policy at the 2017 British Medical Association junior doctors conference requesting training for future doctors in ward round conduct, and clarity and guidance regarding the role of FY1s in ward round conduct. ${ }^{14}$ Similar surveys should be repeated over time to assess the impact of changes in demand and staffing within the NHS on FY1-led ward rounds.

\section{Conclusion}

Newly graduated doctors in the UK are regularly conducting medical ward rounds alone from as early as their first rotation, 
but few receive specific training in this area and most do not feel prepared or supported in doing so. Further research could explore how the involvement of newly graduated doctors in conducting medical ward rounds in the UK compares with other specialties and other countries, and whether this has any effect on patient care. It would also be interesting to see how this varies between regions and from DGHs to tertiary hospitals. Given the frequency with which FY1s are undertaking ward rounds alone, more work is urgently required to prepare future doctors for their role in conducting ward rounds and to ensure they are supported in doing so.

\section{References}

1 Royal College of Physicians. Ward rounds in medicine: principles for best practice. London: RCP, 2012. Ward rounds in medicine: principles for best practice. www.rcplondon.ac.uk/file/1398/ download?token=Zi6E3zx_ [Accessed 28 June 2018].

2 Nikendei C, Kraus B, Schrauth M et al. Ward rounds: how prepared are future doctors? Med Teach 2008;30:88-91.

3 Powell N, Bruce CG, Redfern O. Teaching a 'good' ward round. Clin Med 2015;15:135-8.

4 General Medical Council. Outcomes for Graduates. GMC, 2015. www.gmc-uk.org/static/documents/content/Outcomes_for_ graduates_Dec_16.pdf [Accessed 28 June 2018].

5 The Foundation Programme. The UK Foundation Programme Curriculum. The Foundation Programme, 2012. www.foundationprogramme.nhs.uk/download.asp?file=FP_Curriculum_2012_WEB_ FINAL.PDF [Accessed 1 June 2018].
6 Pearce J, Redman R, Gajebasia S et al. Ward rounds: can an aide-memoire help new doctors? Clin Teach 2018.

7 Morgan J, Green V, Blair J. Using simulation to prepare for clinical practice. Clin Teach 2017;15:57-61.

8 The Foundation Programme. Recruitment stats and facts. The Foundation Programme, 2016. www.foundationprogramme.nhs. uk/download.asp?file=stats_and_facts_fp2016_interim_report_ april_2016_v4.pdf [Accessed 28 June 2018].

9 Likert R. A technique for the measurement of attitudes. Arch Psychol 1932;140:5-55.

10 Harvey R, Mellanby E, Dearden E, Medjoub K, Edgar S. Developing non-technical ward- round skills. Clin Teach 2015;12:336-40.

11 Krautter M, Koehl-Hackert N, Nagelmann L et al. Improving ward round skills. Med Teach 2014;36:783-8.

12 Morgan J, Green V, Blair J. Using simulation to prepare for clinical practice. Clin Teach. 2018;15:57-61.

13 Teagle AR, George M, Gainsborough N, Haw I, Okorie M. Preparing medical students for clinical practice: easing the transition. Perspect Med Educ 2017;6:277-80.

14 The British Medical Association. Junior doctors conference agenda and guide. BMA, 2017. www.bma.org.uk/-/media/files/pdfs/events/2017/ may/jdc-conference-agenda-2017.pdf [Accessed 28 June 2018].

Address for correspondence: Dr Jessica Pearce, Hull York Medical School, John Hughlings Jackson Building, University of York, Heslington, York, Y010 5DD, UK.

Email: hy9jp1@hyms.ac.uk

\section{Assessing trainees in the workplace}

\author{
An e-learning module for \\ secondary care doctors
}

\author{
Three hours of CPD-approved \\ interactive learning covering: \\ $>$ feedback \\ $>$ supervised learning events (SLES) \\ $>$ workplace-based assessments \\ (WPBAs) \\ $>$ the role of the Annual Review of \\ Competence Progression (ARCP).
}

For more information please visit: www.rcplondon.ac.uk/elearning

\section{Royal College of Physicians}



